\title{
The Application of Humanistic Teaching Methods in English Teaching
}

\author{
Shengliang Wang \\ Zhejiang Yuexiu University, Shaoxing, China \\ Email:20051013@zyufl.edu.cn
}

How to cite this paper: Wang, S.L. (2021) The Application of Humanistic Teaching Methods in English Teaching. Open Access Library Journal, 8: e7650.

https://doi.org/10.4236/oalib.1107650

Received: June 16, 2021

Accepted: August 1, 2021

Published: August 4, 2021

Copyright () 2021 by author(s) and Open Access Library Inc.

This work is licensed under the Creative Commons Attribution International License (CC BY 4.0).

http://creativecommons.org/licenses/by/4.0/

\section{(c) (i) Open Access}

\begin{abstract}
Humanistic teaching method can promote the cognitive development of students, but more importantly, it can develop emotional education for students. This article will briefly introduce the basic rationales and principles of the humanistic teaching method, teaching methods based on humanism and teaching activities that embody humanistic ideas, so as to explore the role of students' emotional factors in promoting cognitive development.
\end{abstract}

\section{Subject Areas}

Language Education

\section{Keywords}

Humanism, Humanistic Teaching Method, English Teaching, Affective Factors

\section{1. 导语}

20 世纪 70 年代, 随着建构主义的出现及由此产生的社会互动理论, 植 根于埃里克森、罗杰和马斯洛思想的人文主义开始渗透和影响第二语言教学 和学习领域。根据其理论, 教育的接受者首先被认为是人, 然后才是学习者。 王桂莲(2005) [1]认为, “如果一个人不能满足其生理和心理上的基本需求, 他可能无法全心全意地专注于语言学习。情感不仅是人的基本需要, 而且是 其他身心活动的条件和前提”。

阿洛尼 (2007) [2] 认为, 与以前忽视不守规矩的学生所受的身体或心理差 辱的传统威权教育不同, 人文主义教育致力于营造一种社会和学术氛围, 保 护学生免受智力压迫、体罚和差辱。根据人文主义思想, 人的独特尊严存在 于他们的创造性思维、批判思维、道德敏感性、自主意志和独特个性中, 因 此人文主义教育必须优先考虑人的尊严价值, 而不是其他任何经济、宗教、 
民族主义或意识形态的价值观。

莫斯科维茨(1978)认为(转引自史蒂维克, 1990) [3], 如今一个教育领域 正在受到关注, 其传播与个人发展、自我接纳和他人接纳有关, 换句话说, 其目的是让学生越来越人性化。因此, 人文主义教育感兴趣的是全人教育, 包括智力层面和情感层面的教育。它与所人文主义心理学、人类潜力发展的 关系最为密切。

梅普斯(1979) [4]认为, 人文教育的目标是超越认知和智力教育, 让全人 教育成为现实, 它关注个人成长和创造力的成长, 并在一定程度上关注自我 导向学习。教育的目标与心理治疗的目标相同: 成就一个全功能的人。一个 能够学习和适应变化的人应包含如下特征：对经验的接受度、灵活性、适应 性以及具有将有机体作为行为基础的信念。

邹心胜(2013)认为[5], 人文主义教育对英语教学的性质产生了巨大影响。 在教学目标上, 英语教学把对人格世界的培养作为重中之重, 优先考虑受教 育对象独立人格和独特个性的形成。在教学方法上, 英语教学强调直觉、意 志等非理性因素和心理状态。如同其他教学形式一样, 外语教学也是培养人 与人、心与心、精神与灵魂之间互动的教育活动。正如雅斯贝尔斯(1992)所言 [6], “所谓教育, 是人对人的主体间灵肉交流, 尤其是老一代对新一代包括 对知识的传授、生命内涵的领悟、意志行为的规范, 并通过文化的传递功能 将文化遗产传给年轻一代, 使他们自由的生长, 并启迪天性。” 我国学者徐 超富(2011)也说 [7], “但凡一项活动只要对人的灵魂有触动, 人性有向善, 思想有启迪, 觉悟有提升, 行为有向美, 知识有长进, 思维有变化, 能力有 发展, 体能有改进, 我们认为这项活动就产生了积极作用, 发生了积极影响, 产生了教育效果，具有教育性”。

黄彩丽(2018)认为 [8], 在英语教学中融入人文主义会给学生及英语教学 带来如下的积极作用: 1) 能够加强学生在自主学习和实践方面的能力, 改善 学生在英语学习上的积极性与主动性; 2) 能够突出学生在教学活动中的主体 作用, 将学生作为课堂的主体, 极大地改变以前满堂灌的教学模式; 3) 能够 构建课堂教学中和谐的师生关系, 增进师生之间的互动、相互理解和相互尊 重; 4) 能够促进学生各方面的发展, 挖掘学生内在潜能, 提高学生综合素质。

综上所述, 人文主义教育给语言教育领域带来了重大变化: 重新定义了 教师和学习者的角色, 优先考虑了学习者的需求, 并使语言教学法发生了重 大变化。

\section{2. 人文主义教育的基本原理和基本原则}

盖奇和伯利纳(1991)认为(转引自阿洛尼, 2007) [2], 人文主义包含三个 主要原则: 个人自我价值、感觉与事实同等重要以及个人、社会和道德发展 与学术发展同等重要。关于第一条原则, 盖奇和伯利纳(1991) [2]指出, 对学 生价值的强调在于意识到他们作为独特的人的尊严和权利。其次, 如果学生 的感受和愿望获得尊重, 其学习的情感因素也会被调动起来, 学生就能形成 积极的自我概念, 进而发展其自我效能。

然而, 有学者认为人文主义过分强调学生的情感, 忘记了认知发展才是 
学习的重中之重。阿诺德(1998)在回应这一批评时指出[9], 要优化语言学习, 必须对已经存在的认知集中度施加影响, 这不是对学生认知发展标准的降低, 而是教师意识到有时选择关注学习中的情感因素是有益的。

莫斯科维茨(1978)认为(转引自史蒂维克, 1990) [3], 青少年在语言学习 的过程中会探索自身定位并寻求自我接纳, 而在此过程中他们往往会感受到 封闭和脱离。人文主义教育则关注个人成长、自我接纳以及他人接纳, 换句 话说, 人文主义教育使学生更加人性化。在莫斯科维茨看来, 人文主义教育 与人文主义心理学和人的潜能发展之间的关系相当密切。

针对人文主义教育，人们可能会问：人文主义教育如何实现教育的人性 化和发现学生的潜力? 莫斯科维茨(1978)认为(转引自史蒂维克, 1990) [3] 这 体现在两个方面: 首先是培养学生的情感, 人文主义教育重视学生学习中的 自我感觉。其次是培养学生的个性, 即培养学生去发现表面行为背后的真实 自我。

除了莫斯科维茨, 其他语言学家也谈到了人文主义和人文主义教育。迈 杰什(1986) [10]认为, 在人文主义心理学方法和交际教学法中, 学习者不是单 纯的语言教学对象, 而是尊严、思想、需求、情感都得到尊重的个体。外语 教师应该助力学习者的自我实现。

瑞弗斯(1983) [11]谈到了在约翰 - 杜威领导下的进步主义教育时代脱颖 而出的人文主义教学思想。她认为, 在 20 世纪 70 年代的个性化运动中, 人 文主义教育注重学习者的个性。在强调人文主义思想的课堂中, 学生不仅能 学到知识, 他们还更愿意谈论自己, 对他人敞开心扉, 表达自己的思想。受 过这种教育的人能够接受新想法, 尝试新事物, 但不会被时髦和肤浅的想法 冲昏头脑, 因为他们能坚持自己的立场。

综上所述, 莫斯科维茨 (1978) (转引自凯斯・约翰逊及海伦・约翰逊, 1998) [12]列出了如下人文主义教育的基本原理和基本原则:

- 人文主义教育的主要目的是为学生提供学习情境以促进学生潜力的充分 发挥。

- 人文主义教育应该同等注重认知教育和情感教育。

- 要使学习变得有意义, 应该认识到情感因素的重要性并将其运用到学习 中。

- 学生应该自己来发现值得学习的知识。

- 每个人都希望发挥他们的潜力。

- 与其他同学保持和谐的关系更有利于学习。

- 了解自己是学习的动力。

- 提高自尊是学习的激励因素。

\section{3. 基于人文主义的教学法}

\section{1. 暗示法}

20 世纪 80 年代, 保加利亚心理学家格雷戈里 ・洛扎诺夫对于学习焦虑 和学习负面感进行了深入研究。洛扎诺夫(2005) [13]认为人们对于自己表现的 
不自信和和对于失败的恐惧感极大阻碍了大脑本身无限学习潜力的开发。为 了让人们摆脱这种不自信和恐惧感, 洛扎诺夫提出了 “暗示法” 理论, 他认 为像古典音乐、艺术、放松、冥想、花朵、多彩环境、奇思妙想、富有戏剧 性的声音、幽默、母语、新名字等要素都会给大脑带来积极的暗示作用, 但 是这些要素不是单独起作用而是需要结合其中几个要素一起发挥作用, 因为 大脑不会对于其中单独的要素做出回应。

一些反对者认为暗示法是有害的, 因为它涉及催眠作用。因为暗示法作 用于人的潜意识，所以人们常说该方法涉及催眠，而这会对人类产生负面影 响。洛扎诺夫强烈否认这一点, 并声称该方法不使用以下技术：催眠、神经 语言规则、呼吸练习、 $\alpha$ 脑电波、特定饮食等[14]。

沈平等(2016)认为 [15], 暗示法的最大优点是它可以利用不同的教室装饰 让学生在学习过程中处于放松状态, 以便达到更好的学习效果。这些教室装 饰可以是海报、鲜花甚至是水族箱。以使用海报为例, 学生即便只是环顾教 室里的海报也能始终保持学习状态, 把注意力放在所学的知识上。而暗示法 的缺点之一是其在不发达国家很难实践, 因为这些国家的学校一个班级普遍 包含 30 40 个学生, 在这样的班级中教师就很难控制所有学生的注意力并开 展个性化教学。缺点之二是这种方法的成本会很高, 在贫穷的学校或国家很 难实行。

暗示法的原理之一是 “音乐会场中假消极状态的学习”。洛扎诺夫认为 音乐可以提高人们的自信心, 用音乐独特的无形力量可以使学生放松, 同时 也可以加深对所学材料的掌握。在教学中应该选用何种音乐可根据情况而定, 但一定要用能达到预期效果的音乐(洛扎诺夫建议一分钟 60 拍的慢速乐章) [13]。崔艳丽(2009)在其对艺术生类学生授课的过程中试验过这种方法。当她 在授课过程中播放精心挑选的音乐时, 学生就会逐渐进入一种放松且愉悦的 状态。她也在授课过程中基于一些艺术生的专业特性，让一些性格外向的学 生表演唱歌和舞蹈。比如她在讲授 Welcome the New Year 这一单元时, 在课 上选取了可以让全体同学合唱的苏格兰民歌《友谊地久长天》，并请一些活 跃的同学来伴舞, 这样的授课方式不仅活跃了课堂气氛, 而且在学生喜闻乐 见的形式中传授了该单元的知识点 [16]。

\section{2. 团体语言学习法}

20 世纪 70 年代, 教育学家查尔斯・柯伦提出了团体语言学习法。在典 型的团体语言教学课堂中, 学习者围坐成一圈。老师站在圈子外面, 告诉他 们这节课的学习目的, 学生需要自己决定学习内容。学生用母语进行发言, 老师用外语进行翻译。学生和老师说的话都会保存为音频文件和文本文件。 之后学生自己听录音, 读文本, 反思自己的学习, 并与同伴和老师进行自由 讨论。

团体语言学习法比较典型地体现了人文主义教学法的特点, 其最大特色 是完全打破了以教师为中心的老传统, 充分发挥学生的主体作用, 重视人的 主观因素和学习心理特点, 注意建立良好的学习环境和人际关系, 最大限度 地发挥学生的主动性, 让学生自己决定学习的内容、方式, 因势利导随机应 
变, 弥补教师指导作用发挥不够这一不足[17]。

\section{3. 默示教学法}

默示教学法是由数学教育设计师凯莱布 - 加特尼奥在 20 世纪 70 年代初 期提出的一种教学法, 它是一种有趣且新颖的教学法, 其关键词是 “默示” 、

“意识”、“自身标准” 和 “参与” (黛安・拉森 - 弗里曼, 1986) [18]。在运 用默示教学法的语言课堂中, 老师大多数时候始终保持沉默并给出非语言线 索, 其教育理念是让学生在语言学习时具有更多的独立性、自主性和责任感, 从而让学生能够使用目标语言表达他们的思想、看法和感受, 并且能够解放 自我(黛安・拉森 - 弗里曼, 1986) [18]。教师应该鼓励学生学习时不必担心犯 错, 因为错误本身就是学习过程的自然组成部分。

“默示” 是默示教学法的关键词之一, 因为该教学法的前提是教师在课 堂上应尽可能保持沉默, 以鼓励学习者 “尽可能多地输出目标语言” [19]。默 示教学法专注于培养学生的创造力、发现问题的能力、解决问题的能力和使 用工具的能力。教师的角色只在于给予最少的指令和纠正, 而在大部分时间 保持沉默, 让学习者努力解决语言问题并 “掌握其机制” [20]。帕蒂尔 (2014) 认为 [21], 教师在默示教学法中使用默示可以达到多种目的: 集中学生的注意 力, 引起学生的反应, 并鼓励他们纠正自己的错误。尽管教师们经常保持沉 默, 但仍处于活跃状态: 教师会用嘴型和手势等技巧来帮助学生发音, 也会 鼓励学生帮助他们的同伴, 进行同伴学习。

\section{4. 全身反应教学法}

由亚瑟(1997) [22]创造的 “全身反应教学法” 的特征从其名称中就可见一 斑: 它结合了语言、身体动作和负责学习的右半脑。亚瑟在语言课中注意到 学生过度的焦虑, 所以他希望创造一种无压力的学习方法来使学生拜托焦虑, 从而能够爱上语言学习。全身反应教学法基于这样的前提: 就像贞儿通过身 体动作回应父母的话来学习母语, 学生也可以通过相同的方式学习第二语言 或外语。老师扮演家长的角色, 帮助学习者获得学习动力并增强他们的自信 心。

魏星(2014)认为[23], TPR 教学法最显著的特点是 “听指令一一做工作”, 即教师在课堂上用目标语发出指令, 这些指令用语往往是祈使句, 例如: 起 立、坐下、跟我一起说、声音响一点、过来这边、回去、看黑板、听磁带、 站成一列/一行、从桌上拿起三角板给我、快速走到门口、快速坐下并大笑等 等。在给出指令的同时, 教师也可做相关动作来让学生明白指令的含义。在 重复指令多次之后, 学生即会理解目标语指令的含义, 这时再让学生自己用 动作演示指令的含义, 学生就可以把动作和指令很好地联系起来, 从而达到 教师所要达到的教学目标。然而杜建勇(2011)指出 [24], 教师在 TPR 课堂中给 出的指令一般比较简单, 其所涉及到的目标语也很简单, 这种方式并不适合 较深内容的教授。所以 TPR 课堂只适合学生初级的听说能力的培养而不能对 学生的读写能力产生较好的促进作用。许多教师往往用 TPR 课堂操练听说, 极少把这种授课技术用于读写的教学。 


\section{4. 体现人文主义思想的教学活动}

多种体现人文主义思想的教学活动可以运用到英语课堂教学中。以下活 动将帮助教师在舒适的氛围中激励害差的学生。学生可成对或分组来开展这 些活动。为了让学生处于放松的状态, 教师也允许学生犯一些小错误。在这 些活动后, 学生将学会如何尊重同学和互相帮助。

\section{1. 打招呼}

对于老师来说, 记住学生的名字非常重要。当老师叫出学生的名字时, 学生会感觉更亲近并有班级归属感。为了更加容易地记住学生的名字, 老师 可以制作带有学生照片的点名册, 点名的时候对照着照片可以让老师快速地 记住学生的名字。

黄绍业(1998) [25]认为, 人们可以用必要的言行来增加积极的行为。那么 教师如何帮助学生创造积极的行为? 教师需要说 “请” 和微笑。教师对学生 的积极态度会让学生摆脱学习的压力和焦虑。黄绍震(1998) [25]还认为, 微笑 就像是别人工作做得好时的拍背鼓励或者给别人的一个拥抱。如果老师始终 以微笑面对学生, 学生就可以在舒适的氛围中学习英语, 而微笑对老师来说 是很轻而易举的事情。

\section{2. 使用引语}

使用引言是开始一堂课的好方法, 也能在课堂上很好地激励学生。教师 可以选择来自电影剧本、小说、杂志、歌曲或名人的各种引语。在教师的引 导下, 学生思考这些引语的含义并从中学到有用的表达。学生也可以使用引 语进行句型练习。许多学生喜欢记忆有趣的引语, 这可以使他们对英语学习 产生兴趣并帮助他们学习英语。

\section{3. 课堂讨论会}

教师可以就学生正在学习的科目或内容举办课堂讨论会。课堂讨论会的 人数以 20 人以下为宜。教师可根据学生前一段时间学习的内容提出问题, 在 讨论会期间也可使用计时器让学生与不同的同学参与讨论。老师可以每三到 五分钟发出交换同学的信号。这样, 学生可以尽可能多地与不同的同学分享 他们的想法。

在课堂讨论会期间, 学生与不同的同学分享他们对某些问题的想法。教 师可播放合适的背景音乐营造课堂讨论会的氛围。通过这种方式, 学生将学 习如何在舒适轻松的氛围中分享他们的想法。问题示例如下:

1) 你叫什么名字? 你名字的含义是什么?

2) 你如何用四个形容词来形容自己?

3）你喜欢怎样度过你的空闲时间?

4) 你能很好地做什么? 你的强项是什么?

\section{4. 情绪温度计}

莫斯科维茨(1978) [26]在人文主义课堂中引入了情绪温度计的概念, 它可 
以帮助教师了解学生的心情, 学生也能学会如何相互尊重。有的同学可能因 为各种原因没有好的心情。然而, 通过使用人文主义教学法, 教师可以让学 生的心情从坏变好, 这就是在课堂上使用情绪温度计的目的。

\section{5. 音乐相伴下的最爱时刻}

音乐对我们的生活有着巨大的影响, 也能让我们思考很多事情。教师可 以在播放舒缓音乐的同时询问学生他们最喜欢的时刻。教师可以先分享他/她 最喜欢的时刻, 给学生一些想法。教师也可让学生带上他们最喜欢的音乐在 课堂上播放, 这会有效地激励学生, 并给学生带来更好的课堂参与感。

\section{6. 牢记 “我能做到” 原则}

每当学生对自己的学习能力感到失望时, 教师可让学生牢记一些增强自 己积极性的句子来鼓励自己, 这可以激发学生学习中积极的情感因素, 对英 语学习非常重要。这些句子来自丽贝卡・奧克斯福德(1990)的著作《语言学习 策略: 每个教师都应该知道的》[27]。

- 老师今天说的几乎所有内容我都能听懂。

- 我今天犯错时没有气馁, 我坚持认真学习。

- 今天我鼓起勇气使用了一个新的表达方式, 我很高兴我做到了。

- 以前我在英语课上不敢发言, 我很高兴我今天做到了。

- 我今天用英语进行了非常成功的对话。

- 我是一个很好的语言学习者。

- 我对语言学习充满信心。

- 我的语言学习进展顺利。

- 在语言学习上具有冒险精神, 我感觉不错。

- 每个人都会犯错, 我会从错误中吸取教训。

- 我不必一下子理解所有内容。

- 我能容忍一点点语言学习中的困惑。

- 我热情的性格帮助我学习语言。

- 我能明白大致的意思, 没有必要掌握每一个字。

- 我可以感觉我的流利度在提升。

\section{5. 结语}

如今快速发展的社会给学生带来了学习上的压力和焦虑, 如果教师在教 学中贯彻人文主义思想, 一方面可以减少学生的学习焦虑, 让学生在放松舒 适的环境下进行语言学习, 另一方面也可以提供一种有效的教学和学习方法, 不仅可以构建学生的知识体系, 还可以实现学生自身的潜力。虽然对于人文 主义教学法仍有一些反对的声音, 但是它在实现全人教育方面是独一无二的。

在对人文主义教学法的学习中也让我逐渐意识到作为一名教师该对学生 负起的责任。教师在自己的教学中应该做到仔细、敏感、周到、系统, 才能 让学生体会到学习的真正乐趣。我的文章也只是对人文主义教学法有非常初 浅的了解, 只是对于人文主义教学法的基本原则、以及里面所涉及到的一些 
基本教学法及其在课堂中的运用做了粗浅的论述, 并未对其理论基础和其他 实际的应用做出深入的探讨。希望以后能和对人文主义教学法同样感兴趣的 同行教师一起研究如何在英语教学中更加深入地推进人文主义教学法。未来 研究方向可包括: 一、教师提高专业素质, 丰富教学方式, 更新教学内容, 使课堂语言教学不至于僵化; 二、教师作为课堂教学组织者和学生情绪引导 者角色的平衡者, 既要完成教学目标, 又要缓解学生学习中的压力, 让学生 在轻松无压力的环境中完成语言学习; 三、改善学生的知识结构, 帮助学生 建立起合理的知识体系, 并把课堂教学延伸至课外, 让学生获得校外环境中 的学习机会。

\section{Conflicts of Interest}

The author declares no conflicts of interest.

\section{References}

[1] Wang, G. (2005) Humanistic Approach and Affective Factors in Foreign Language Teaching. Sino-US English Teaching, 2, 1-5.

[2] Aloni, N. (2007) Enhancing Humanity. Springer, Dordrecht.

[3] Stevick, E.W. (1990) Humanism in Language Teaching: A Critical Perspective. Oxford University Press, Oxford.

[4] Maples, M.F. (1979) A Humanistic Education: Basic Ingredients. The Humanistic Educator, 17, 107-110.

[5] 邹心胜. 大学外语教育的理论与实践探索一一基于人文主义视角 [D]: [博士学位 论文]. 武汉: 华中师范大学, 2013.

[6] 雅斯贝尔斯. 什么是教育 $[\mathrm{M}]$. 上海: 三联书店, 1992.

[7] 徐超富. 大学科学研究之教育意蕴 [J]. 湖南师范大学教育科学学报, 2011, 10(1): 20-27.

[8] 黄彩丽. 英语教育应注重人文主义的融入 [J]. 产业与科技论坛, 2018，17(3), 152-153.

[9] Arnold, J. (1998) Towards More Humanistic English Teaching. ELT Journal, 52, 235-242.

[10] Medgyes, P. (1986) Queries from a Communicative Teacher. English Language Teaching Journal, 40, 107-112.

[11] Rivers, W. (1983) Communicating Naturally in a Second Language: Theory and Practice in Language Teaching. Cambridge University Press, Cambridge.

[12] Johnson, K. and Johnson, H. (1998) Encyclopedic Dictionary of Applied Linguistics: A Handbook for Language Teaching. Blackwell Publishers, Oxford.

[13] Lozanov, G. (2005) Suggestopaedia-Desuggestive Teaching Communicative Method on the Level of the Hidden Reserve of the Human Mind. http://dr-lozanov.dir.bg/book

[14] Talley, P.C. (2016) A Relative Comparison Made of Two Teaching Methodologies: The Examples of Suggestopedia vs. Total Physical Response. International Journal of Humanities and Social Science, 6, 40-46.

[15] Shen, P., Xue, S.-J. and Zhu, L. (2016) The Characteristics and Methods of Adult Learners. 2nd International Conference on Social Science and Higher Education, Sanya, 23-24 July 2016, 40-43. https://dx.doi.org/10.2991/icsshe-16.2016.27 
[16] 崔艳丽. 暗示法在艺术类英语教学中的应用 [J]. 湖北经济学院学报(人文社会科 学版), 2009, 6(10): 198-199.

[17] 刘珣. 对外汉语教育学引证[M]. 北京: 北京大学出版社, 2010.

[18] Diane, L.-F. (1986) Techniques and Principles in Language Teaching. Oxford University Press, New York.

[19] Cook, V. (2008) Second Language Learning and Language Teaching. Arnold, London.

[20] Bruner, J. (1966) Toward a Theory of Instruction. Harvard University Press, Cambridge, MA.

[21] Patil, Y.R. (2014) The Silent Way and Other Two Methods of Language Teaching. Online International Interdisciplinary Research Journal, 4, 285-287.

[22] Asher, J. (1997) The Jackendoff "Skeptic" on Humanistic Language Teaching. http://www.htmag.co.uk

[23] 魏星. TPR 教学法在初级汉语口语课堂教学中的应用研究[D]: [硕士学位论文]. 西安: 西安外国语大学, 2014.

[24] 杜建勇. TPR 教学法在对外汉语教学中的应用[D]: [硕士学位论文]. 上海: 上海 师范大学, 2011.

[25] Wong. H.K. and Wong. R.T. (1998) The First Day of School. Harry K. Wong Publications, Inc., California.

[26] Moskovitz, G. (1978) Caring and Sharing in the Foreign Language Classroom. Newbury House Publishers, Inc., New York.

[27] Oxford, R.C. (1990) Language Learning Strategies. Newbury House Publishers, Inc., New York.

\section{Appendix (Abstract and Keywords in Chinese)}

\section{人文主义教学法在英语教学中的应用}

摘要: 人文主义教学法可以促进学生的认知发展, 但更加重要的是它可 以开展对学生的情感教育。本文将简要介绍人文主义教学法的基本原理和基 本原则, 基于人文主义的教学法和体现人文主义思想的教学活动, 从而来探 索学生的情感因素对认知发展的促进作用。

关键词: 人文主义; 人文主义教学法; 英语教学; 情感因素 